\title{
Treatment Options and Outcomes of Vancomycin-Resistant Enterococcus in Prosthetic Joint Infections: Case Report and Potential Implications for Military Cases
}

\author{
Natalia I. Sidhu, ${ }^{1}$ and Suresh J. Antony ${ }^{1,2,}{ }^{*}$ \\ ${ }^{1}$ Paul L. Foster School of Medicine, Texas Tech University Health Sciences Center, El Paso, TX, USA \\ ${ }^{2}$ Burrell College of Osteopathic Medicine, Las Cruces, NM, USA \\ "Corresponding author: Suresh J. Antony, MD, 1205 N. Oregon St., El Paso, TX, USA. Tel: +98-9155334900, Fax: +98-9155334902, E-mail: suresh.antony@att.net
}

Received 2017 February 28; Revised 2017 May 05; Accepted 2017 August 15.

\begin{abstract}
Introduction: Vancomycin-resistant Enterococcus (VRE) infection of prosthetic joints is an unusual infection. When it does occur, it is difficult to treat, as treatment options are limited and often fraught with difficulties.

Case Report: We present a case of a patient with a VRE-infected total knee arthroplasty that was treated with a two-stage revision along with six weeks of intravenous and intraarticular daptomycin with a good outcome.

Conclusions: A protocol for treating VRE in prosthetic joint infections has yet to be established and further randomized controlled studies are warranted for the management of such infections.
\end{abstract}

Keywords: Intraarticular Antibiotics, Prosthetic Joint Infections, Total Knee Arthroplasty, Vancomycin-Resistant Enterococci

\section{Introduction}

Total joint arthroplasty is becoming a commonly used surgery in the ageing population, providing for pain relief and increased mobility. From 2000 to 2009, rates of knee replacement in the United States nearly doubled, increasing from about 120 to 215 per 100,000 people (1). Infection, however, poses one of the greatest risks of failure, found in $0.5 \%$ to $3 \%$ of patients (1-3). Within the veterans health administration, $43 \%$ of readmissions in the year post hip or knee arthroplasty are caused by infection (4). Risk of failure increases substantially if the infection is caused by a multidrug-resistant (MDR) organism, including methicillin-resistant Staphylococcus aureus (MRSA) and vancomycin-resistant Enterococcus(VRE). Failure rates of arthroplasty in cases of MDR bacteria range from $24 \%$ to $82 \%$ (5-8).

While substantial literature exists describing prosthetic joint infections (PJI) caused by MRSA, less has been written covering VRE $(7,9)$. We present a case of VRE affecting a total knee arthroplasty (TKA) that was treated with a two-stage revision along with intravenous and intraarticular daptomycin for a 6-week period with a good outcome.

\section{Case Presentation}

A 72-year-old female with a history of diabetes mellitus, hypertension, and osteoarthritis who underwent a right TKA 3 years prior to presentation was seen by her physician for pain, swelling, and tenderness to the knee. At the time of her examination, her temperature was 100.2F, blood pressure was 124/76 mm Hg and pulse was 96 bpm. Pertinent findings on exam included an ill-appearing woman with an edematous knee and significant tenderness to the joint. There was also evidence of a synovial effusion. The remaining physical examination was unremarkable. Laboratory findings were significant for an erythrocyte sedimentation rate (ESR) of $56 \mathrm{~mm} / \mathrm{h}$ and C-reactive protein (CRP) of $5.6 \mathrm{mg} / \mathrm{dL}$.

Arthrocentesis of the affected joint revealed turbid synovial fluid with a leukocyte count of 34.202 cells $/ \mathrm{mm}^{3}$ and red blood cell count of 124 cells $/ \mathrm{mm}^{3}$. Cultures of the aspirated fluid were negative. She was taken to surgery and underwent an incision and drainage with a change of the polymer and subsequent treatment with ceftriaxone $2 \mathrm{~g}$ daily for 6 weeks. Despite the prolonged course of antibiotics, she continued to have pain and persistent elevation of her ESR (68 mm/h) and CRP (4.4 mg/dL).

Eight months after the incision and drainage, she was taken back to surgery for a two-stage revision of the knee, upon which synovial fluid cultures showed heavy growth of VRE. At this time, she was clinically ill-looking. Her temperature was 101.1F, blood pressure $98 / 78 \mathrm{mmhg}$, and pulse $102 \mathrm{bpm}$. The knee was swollen, tender, and drained serosanguineous fluid. A Hickman catheter was placed in the joint, and the patient was administered $100 \mathrm{mg}$ daptomycin daily in 2 cc saline intraarticularly as well as 4 
$\mathrm{mg} / \mathrm{kg} /$ day intravenously. This was done for a total of six weeks. Serial cultures from the Hickman of the synovial fluid did not show regrowth of the VRE. Four weeks into treatment, ESR and CRP were $24 \mathrm{~mm} / \mathrm{h}$ and $2.3 \mathrm{mg} / \mathrm{dL}$, respectively. Follow-up four months later revealed normalization of the ESR and CRP with no evidence of persistence of the infection.

\section{Discussion}

Overall lifetime infection rates of prosthetic joints are up to $3 \%$ (1-3). Current options for treating PJI include open debridement and reimplantation in either one or two stages (8-10). In addition to surgical intervention, intravenous antibiotics should be administered for approximately six weeks $(6,8,9)$. After an initial infection, average failure rate of arthroplasty ranges from $10 \%$ to $20 \%$, depending on the pathogen $(2,10,11)$. However, when the prosthesis is infected with a resistant organism, these rates increase up to 4 -fold $(8,9)$.

An estimated 3\% to 10\% of PJI by Gram-positive bacteria are caused by Enterococcus spp. (2). Morbidity and mortality of these infections are higher than with other organisms. One proposal for this is that nearly half of all patients with VRE infections have at least one comorbidity, including obesity, diabetes mellitus, coronary artery disease, or chronic kidney failure $(2,12)$.

VRE infections were first reported in hospital illnesses in Great Britain in $1988(13,14)$. Since then, increased rates of these resistant infections are due in part to factors such as overuse of vancomycin in a variety of clinical settings, advances in and increased invasiveness of surgical procedures, the general population's extended life expectancy, and improved survival of patients with immunodeficiency (14). Nine phenotypes of VRE have been characterized to date, leading to resistance caused by both phenotypic and genotypic variation (14). Resistance to vancomycin is achieved by receptors with reduced affinity for glycopeptides, resulting in decreased antibiotic binding and ultimately decreased inhibition of bacterial cell wall synthesis $(14,15)$.

Due to the difficulty in treating VRE, a two-stage approach has traditionally been recommended for resistant Enterococcal joint infections. This promotes eradication of infection and restoration of the joint's function $(2,8)$. With such a procedure, an interim cement spacer is implanted until a new prosthesis may be inserted (9). Although these spacers initially allow for high concentrations of intraarticular antibiotics, local concentrations decrease after 3 to 7 days $(9,16)$. Decreased antibiotic elution over time may allow for growth of resistant bacteria, which require sus- tained exposure to high concentrations of antibiotic for eradication $(9,17)$.

In addition to surgical management, administration of intravenous antibiotics, such as daptomycin, has also been utilized in PJI. Daptomycin has been shown to have bactericidal activity in treating Gram-positive orthopedicrelated infections at high doses $(4-6 \mathrm{mg} / \mathrm{kg} / \mathrm{day})(12,18)$. While substantial reports exist on its use in PJI caused by MRSA and non-resistant Enterococcus, daptomycin has been used infrequently to treat VRE-associated PJI $(12,18)$.

A relatively novel approach to overcome spacers' limited ability to sustain high concentrations of intraarticular antibiotics involves the placement of catheters within the joint. This allows for localized delivery of antibiotics, thereby increasing penetration into the joint space $(3,9)$. A one-stage revision with placement of Hickman catheters allows for higher rates of microbiologic cure, characterized by negative follow-up synovial fluid cultures in patients with susceptible pathogens (3). Most significantly, the use of catheters supplying intraarticular antibiotics allows possible improvement of arthroplasty salvage rates and less limb loss, as compared to more traditional twostage revision (3,19-21).

High levels of antibiotic may overcome the resistance mechanisms of the bacteria, and thus the minimum inhibitory concentration, without causing systemic toxicity $(3,20,21)$. The concentrations in adjacent bone are also higher via intraarticular antibiotics as compared to intravenous administration $(9,20,21)$. Specifically, intraarticular daptomycin has been used successfully once to our knowledge for VRE-associated PJI (3). Intraarticular use of other antibiotics such as linezolid and quinupristindalfopristin is not yet available in the literature.

In our case, intraarticular and intravenous daptomycin were used to treat PJI caused by VRE. Advantages of this method include the bactericidal action of daptomycin and ease of antibiotic administration. Disadvantages are that clear dose recommendations are not yet available for treating infections using an intraarticular route. Longterm follow-up for VRE-associated PJI are available, but are small in number (Table 1). These cases are too few to make definitive recommendations.

In conclusion, while joint arthroplasty can vastly improve the quality of life of the patient, complications related to infection need to be minimized to ensure adequate benefit of such procedures. Upon reviewing the literature, a protocol for treating VRE in PJI has yet to be established. Moreover, antibiotics not approved for use in treating VRE have been utilized in the past, indicating the need for such a protocol (11). Notably, a prospective study of over 18,500 cases of total joint arthroplasty within the veterans health administration showed that as compared to their high- 
Table 1. Treatment and Outcomes of vancomycin-Resistant Enterococcus-Associated Prosthetic Joint Infection

\begin{tabular}{|c|c|c|c|c|c|c|c|}
\hline Study & Year & Number of Knees & Number of Hips & $\begin{array}{l}\text { Underlying } \\
\text { Disorders }\end{array}$ & $\begin{array}{l}\text { Antibiotic } \\
\text { Regimen }\end{array}$ & $\begin{array}{c}\text { Number of } \\
\text { Resolved } \\
\text { Infections }\end{array}$ & $\begin{array}{c}\text { Mean Follow-Up, } \\
\text { mo }\end{array}$ \\
\hline $\begin{array}{l}\text { Whiteside and } \\
\text { Roy (21) }\end{array}$ & 2016 & 1 & 0 & CAD & $\begin{array}{l}\text { IV daptomycin } 6 \\
\mathrm{mg} / \mathrm{kg}+\mathrm{IV} \\
\text { ampicillin x } 6 \mathrm{w}\end{array}$ & 1 & 0.25 \\
\hline Antony et al. (3) & 2015 & 1 & 0 & DM & $\begin{array}{l}\text { IA daptomycin } \\
\text { (100 mg daily) }\end{array}$ & 1 & 6 \\
\hline Till et al. (22) & 2002 & 0 & 1 & $\mathrm{OA}$ & $\begin{array}{l}\text { PO linezolid } 600 \\
\text { mg BID x } 8 \mathrm{w}\end{array}$ & 1 & 12 \\
\hline Ries (11) & 2001 & 2 & 0 & $\begin{array}{l}\text { DM, angina, HTN, } \\
\text { asthma; hepatitis }\end{array}$ & $\begin{array}{l}\text { IV } \\
\text { chloramphenicol } 1 \\
\text { g q } 6 \text { h x } 3 \mathrm{w} ; \text { PO } \\
\text { doxycycline } 100 \\
\text { mg BID x } 6 \text { mo }\end{array}$ & 2 & 9 \\
\hline
\end{tabular}

Abbreviations: CAD, coronary artery disease; DM, diabetes mellitus; IA, intraarticular; IV, intravenous; OA, osteoarthritis.

income and male counterparts, low-income veterans and women are more likely to develop major complications after joint replacement, including infection of the prosthesis (4). Thus, this population of veterans is at especially highrisk and would benefit from such treatment protocols.

Moreover, synergistic combinations of antibiotics are a further possibility in treating these resistant infections. Success has been shown with the use of daptomycin combined with ampicillin, and in vitro studies with linezolid combined with minocycline show promise $(14,23)$. Nevertheless, randomized controlled studies are needed to establish guidelines for surgical and pharmacologic management of VRE in PJI.

Implication for health policy/practice/research/medical education: to our knowledge, we present the first case of intraarticular daptomycin being used successfully to treat total knee arthroplasty infected with vancomycin-resistant Enterococcus.

\section{References}

1. Lamagni T. Epidemiology and burden of prosthetic joint infections. J Antimicrob Chemother. 2014;69 Suppl 1:i5-10. doi: 10.1093/jac/dku247. [PubMed: 25135091].

2. Tornero E, Martinez-Pastor JC, Bori G, Garcia-Ramiro S, Morata L, Bosch J, et al. Risk factors for failure in early prosthetic joint infection treated with debridement. Influence of etiology and antibiotic treatment. J Appl Biomater Funct Mater. 2014;12(3):129-34. doi: 10.5301/jabfm.5000209. [PubMed: 25199072].

3. Antony SJ, Westbrook RS, Jackson JS, Heydemann JS, Nelson JL. Efficacy of Single-stage Revision with Aggressive Debridement Using Intra-articular Antibiotics in the Treatment of Infected Joint Prosthesis. Infect Dis (Auckl). 2015;8:17-23. doi: 10.4137/IDRT.S26824. [PubMed: 26279625].

4. Weaver F, Hynes D, Hopkinson W, Wixson R, Khuri S, Daley J, et al. Preoperative risks and outcomes of hip and knee arthroplasty in the Veterans Health Administration. J Arthroplasty. 2003;18(6):693-708. [PubMed: 14513441].
5. Whiteside LA, Nayfeh TA, LaZear R, Roy ME. Reinfected revised TKA resolves with an aggressive protocol and antibiotic infusion. Clin Orthop Relat Res. 2012;470(1):236-43. doi: 10.1007/s11999-011-2087-4. [PubMed: 21948323].

6. Mittal Y, Fehring TK, Hanssen A, Marculescu C, Odum SM, Osmon D. Two-stage reimplantation for periprosthetic knee infection involving resistant organisms. J Bone Joint Surg Am. 2007;89(6):1227-31. doi: 10.2106/JBJS.E.01192. [PubMed: 17545425].

7. Salgado CD, Dash S, Cantey JR, Marculescu CE. Higher risk of failure of methicillin-resistant Staphylococcus aureus prosthetic joint infections. Clin Orthop Relat Res. 2007;461:48-53. doi: 10.1097/BLO.ob013e3181123d4e. [PubMed: 17534195].

8. Vasso M, Schiavone Panni A, De Martino I, Gasparini G. Prosthetic knee infection by resistant bacteria: the worst-case scenario. Knee Surg Sports Traumatol Arthrosc. 2016;24(10):3140-6. doi: 10.1007/s00167-0164010-8. [PubMed: 26831859].

9. Whiteside LA, Peppers M, Nayfeh TA, Roy ME. Methicillin-resistant Staphylococcus aureus in TKA treated with revision and direct intraarticular antibiotic infusion. Clin Orthop Relat Res. 2011;469(1):26-33. doi: 10.1007/s11999-010-1313-9. [PubMed: 20390472].

10. Antony S. Use of Intra-Articular Antibiotics Along with Single Stage Revision to Treat Prosthetic Joint Infections (PJI)/Orthopedic Related Devices (ORD): Suggestions for Selection of the Appropriate Patients for this Procedure. Infect Disord Drug Targets. 2016 [PubMed: 27411472].

11. Ries MD. Vancomycin-resistant Enterococcus infected total knee arthroplasty. J Arthroplasty. 2001;16(6):802-5. doi: 10.1054/arth.2001.24951. [PubMed: 11547381].

12. Antony SJ. Combination therapy with daptomycin, vancomycin, and rifampin for recurrent, severe bone and prosthetic joint infections involving methicillin-resistant Staphylococcus aureus. Scand JInfect Dis. 2006;38(4):293-5. [PubMed: 16718933].

13. Uttley AH, George RC, Naidoo J, Woodford N, Johnson AP, Collins CH, et al. High-level vancomycin-resistant enterococci causing hospital infections. Epidemiol Infect. 1989;103(1):173-81. [PubMed: 2506070].

14. Wu J, Jiang TT, Su JR, Li L. Antimicrobial activity of linezolid combined with minocycline against vancomycin-resistant Enterococci. Chin Med J (Engl). 2013;126(14):2670-5. [PubMed: 23876894].

15. Cooper MA, Fiorini MT, Abell C, Williams DH. Binding of vancomycin group antibiotics to D-alanine and D-lactate presenting self-assembled monolayers. Bioorg Med Chem. 2000;8(11):2609-16. [PubMed: 11092546].

16. Anguita-Alonso P, Rouse MS, Piper KE, Jacofsky DJ, Osmon DR, Patel R. Comparative study of antimicrobial release kinetics from poly- 
methylmethacrylate. Clin Orthop Relat Res. 2006;445:239-44. doi: 10.1097/01.blo.0000201167.90313.40. [PubMed: 16474225].

17. Neut D, Hendriks JG, van Horn JR, van der Mei HC, Busscher HJ. Pseudomonas aeruginosa biofilm formation and slime excretion on antibiotic-loaded bone cement. Acta Orthop. 2005;76(1):109-14. doi: 10.1080/00016470510030427. [PubMed: 15788318].

18. Rice DA, Mendez-Vigo L. Daptomycin in bone and joint infections: a review of the literature. Arch Orthop Trauma Surg. 2009;129(11):1495504. doi: 10.1007/s00402-008-0772-x. [PubMed: 18989686].

19. Peel TN, Cheng AC, Buising KL, Choong PF. Microbiological aetiology, epidemiology, and clinical profile of prosthetic joint infections: are current antibiotic prophylaxis guidelines effective? Antimicrob Agents Chemother. 2012;56(5):2386-91. doi: 10.1128/AAC.06246-11. [PubMed: 22314530].
20. Whiteside LA, Roy ME, Nayfeh TA. Intra-articular infusion: a direct approach to treatment of infected total knee arthroplasty. Bone Joint J. 2016;98-B(1 Suppl A):31-6. doi:10.1302/0301-620X.98B.36276. [PubMed: 26733638].

21. Whiteside LA, Roy ME. One-stage Revision With Catheter Infusion of Intraarticular Antibiotics Successfully Treats Infected THA. Clin Orthop Relat Res. 2017;475(2):419-29. doi: 10.1007/s11999-016-4977-y. [PubMed: 27511201].

22. Till M, Wixson RL, Pertel PE. Linezolid treatment for osteomyelitis due to vancomycin-resistant Enterococcus faecium. Clin Infect Dis. 2002;34(10):1412-4. doi:10.1086/340265. [PubMed: 11981739].

23. Desai H, Wong R, Pasha AK. A Novel Way of Treating Multidrugresistant Enterococci. $N$ Am J Med Sci. 2016;8(5):229-31. doi: 10.4103/1947-2714.183015. [PubMed: 27298819]. 